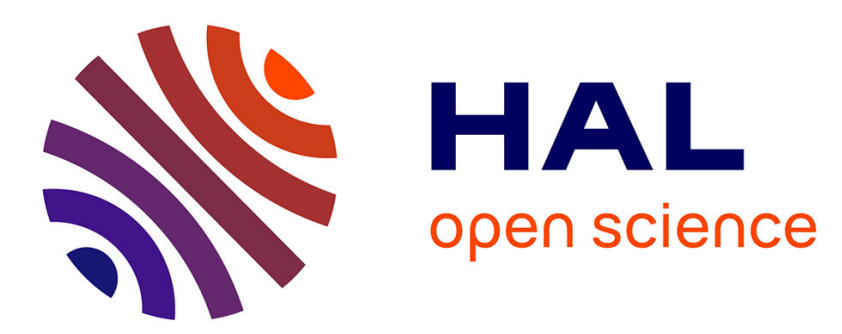

\title{
Circularity of Complex Stochastic Models in POLSAR and Multi-pass InSAR Images
}

Gabriel Vasile, Felix Totir

\section{To cite this version:}

Gabriel Vasile, Felix Totir. Circularity of Complex Stochastic Models in POLSAR and Multi-pass InSAR Images. IGARSS 2012 - IEEE International Geoscience and Remote Sensing Symposium, Jul 2012, Munich, Germany. pp.3720-3723. hal-00785995

\section{HAL Id: hal-00785995 \\ https://hal.science/hal-00785995}

Submitted on 7 Feb 2013

HAL is a multi-disciplinary open access archive for the deposit and dissemination of scientific research documents, whether they are published or not. The documents may come from teaching and research institutions in France or abroad, or from public or private research centers.
L'archive ouverte pluridisciplinaire HAL, est destinée au dépôt et à la diffusion de documents scientifiques de niveau recherche, publiés ou non, émanant des établissements d'enseignement et de recherche français ou étrangers, des laboratoires publics ou privés. 


\title{
CIRCULARITY OF COMPLEX STOCHASTIC MODELS IN POLSAR AND MULTI-PASS INSAR IMAGES
}

\author{
G. Vasile ${ }^{1}$, F.C. Totir ${ }^{2}$ \\ 1: GIPSA-lab, CNRS / Grenoble INP, 961 rue de la Houille Blanche, BP 46, \\ F-38402 GRENOBLE Cedex, France \\ gabriel.vasile@gipsa-lab.grenoble-inp.fr \\ 2: CYBERIO, 6 bis, Chemin des Prés, F-38240 MEYLAN, France \\ felix.totir@cyberio-dsi.com
}

\begin{abstract}
Polarimetry and multi-pass interferometry extend the dimensionality of SAR data, so the necessity to have multivariate statistic (and non-Gaussian, because of the high resolution) distributions as models for these types of data: such are the SIRV (Spherically Invariant Random Vectors). However, as the statistic model becomes so complicated, correctly estimating its parameters gets difficult. More, although they are versatile, the SIRV models are not guaranteed to match the PolSAR / InSAR data. To evaluate the pertinence of those models with respect to the PolSAR data, through one of their most important statistic property, namely the circularity, it is the purpose of this paper.
\end{abstract}

Index Terms - Polarimetry, SAR, circularity, SIRV

\section{INTRODUCTION}

Multidimensional Synthetic Aperture Radar (SAR) data, like PolSAR and multi-pass InSAR images, describe interaction between the electromagnetic waves and the backscatterrers inside each resolution cell, thus allowing extracting information concerning the physical properties of the illuminated target. Generally, and especially for distributed targets (terrains, forests, etc.), the analysis relies on the stochastic properties of the data. The classic approach, assuming Gaussian model, is quite limited and already outdated in the context of improved resolution of modern SAR. Diminishing the size of the resolution cells leads to a reduction on the number of backscatterrers inside each resolution cell. Thus, the central limit theorem is no longer applicable and the Gaussian model must be reconsidered. More complex stochastic models, such as the SIRP (Spherically Invariant Random Process), are then required. Several special cases (K-compound, Weibull, etc.) of those have been already used instead of Gaussian distributions in some specific areas of interest (for example, in coastal radar applications) before being reunited under the common umbrella of SIRP.

For example, polarimetry extends the dimensionality of recorded data (to 3 or 4) and, thus, multivariate versions of SIRP distributions, namely SIRV (Spherically Invariant Random Vectors), are used in PolSAR.

However, the more complex the (stochastic) model, the more difficult estimating its parameters becomes. More, the question of either or not the SIRV models, in spite of their flexibility, are appropriate for describing any multidimensional SAR dataset, still remains. This fact prompted us to analyze, in this paper, the pertinence of those models with respect to various multidimensional SAR datasets, especially in the light of a specific and very important (because often assumed) stochastic property: the circularity.

\section{STOCHASTIC MODELS IN POLSAR}

The four components of the $\mathbf{k}$ target vector describing the polarimetric characteristics of a given resolution cell are the elements of the Sinclair matrix: $S_{h h}, S_{h v}, S_{v h}$ and $S_{v v}$. For mono-static configurations, where the reciprocity theorem applies, $S_{h v}=S_{v h}$, so only three independent components remain: $S_{h h}, S_{h v}$ and $S_{v v}$. In this case, the useful vector becomes $\mathbf{k}=\frac{1}{\sqrt{2}}\left[S_{h h}-S_{v v}, 2 S_{h v}, S_{h h}+S_{v v}\right]^{T}$.

For distributed targets, the corresponding $\mathbf{k}$ vector is considered non-deterministic and its multivariate probability distribution may be written, under the SIRV assumption: $\mathbf{k}=\sqrt{\tau} \mathbf{z} . \tau$ is a positive random variable (texture) while $\mathbf{z}$ is a complex-valued, centered and Gaussian distributed, random vector. The two random variables are statistically independent.

Characterizing $\mathbf{k}$, in this case, reduces to write the probability distribution $p_{\tau}(\tau)$ of $\tau$ and the covariance 
matrix $\mathbf{M}$ of $\mathbf{z}$. If $p_{\tau}(\tau)$ is a Dirac pulse, then the Gaussian (multivariate) model is retrieved for $\mathbf{k}$. In many field applications, $p_{\tau}(\tau)$ is assumed to be the Fisher probability distribution. In this latter case, the obtained SIRV distribution is known as KummerU [1].

In the final paper, this presentation will include the multipass InSAR case.

\section{CIRCULARITY OF COMPLEX RANDOM PROCESSES}

A complex-valued random vector $Z_{0}$ is circular [2], [14] (i.e. its stochastic distribution has circular symmetry) if its distribution remains invariant to multiplication with complex numbers on the unity circle: $Z_{0}$ has the same stochastic distribution as $e^{j \theta} Z_{0}$.

Characterizing a complex-valued random vector $Z_{0}$ simply means characterizing its real and imaginary parts, $X_{0}$ and $Y_{0}$ respectively. Thus, $Z_{0}$ is circular if and only if the real random vector $V_{0}=\left(X_{0}, Y_{0}\right)^{T}$ is spherically symmetric with respect to the origin. This also means that the characteristic function of $Z_{0}$ may be written as $\Phi_{Z_{0}}(z)=\phi\left(|z|^{2}\right)$, where $\phi:[0, \infty[\rightarrow \Re$ is named characteristic generator. If defined, the probability density of $Z_{0}$ takes the form $f_{Z_{0}}(z)=g\left(|z|^{2}\right)$, where $g:[0, \infty[\rightarrow[0, \infty[$ is known as density generator [3].

\section{TESTING THE CIRCULARITY}

Let's consider the mono-static POLSAR case, where the vector has three complex-valued components.

\subsection{Gaussian random processes}

In the Gaussian case, the stochastic model is:

$$
p_{G}(\mathbf{k})=\frac{1}{\pi^{m}|\mathbf{T}|} e^{-\mathbf{k}^{H} \mathbf{T}^{-1} \mathbf{k}}
$$

where $m=3$ (for the mono-static case). Define the covariance $\mathbf{T}=E\left\{\mathbf{k k}^{H}\right\}$, the pseudo-covariance [4], [15] $\mathbf{C}=E\left\{\mathbf{k} \mathbf{k}^{T}\right\}$, the extended vector $\mathbf{z}=\left[\mathbf{k}^{T}, \mathbf{k}^{H}\right]^{T}$ and, finally, the SCM (Sample Covariance Matrix) estimator $\hat{\mathbf{R}}_{S C M}=1 / N \sum_{i=1}^{N} \mathbf{z}_{i} \mathbf{z}_{i}^{H}$, then the sphericity test relies on the GLRT (Generalized Likelihood Ratio Test).
The considered hypotheses are:

1. $\mathrm{H}_{1}: \mathbf{k}$ is spherically, i.e. $\mathbf{C}=\mathbf{0}_{m}$

2. $\mathrm{H}_{2}: \mathbf{k}$ is not spherically, i.e. $\mathbf{C} \neq \mathbf{0}_{m}$

The sphericity test then becomes [5], [6]:

$\Lambda\left(\mathbf{k}_{1}, \ldots, \mathbf{k}_{N}\right)=\frac{\max _{\hat{\mathbf{R}}_{S C M}, \mathbf{C}=\mathbf{0}_{m}}\left\{p_{\mathbf{k}}\left(\mathbf{k}_{1}, \ldots, \mathbf{k}_{N}\right)\right\}}{\max _{\hat{\mathbf{R}}_{S C M}}\left\{p_{\mathbf{k}}\left(\mathbf{k}_{1}, \ldots, \mathbf{k}_{N}\right)\right\}}=\frac{\left|\hat{\mathbf{R}}_{S C M}\right|}{\left|\hat{\mathbf{T}}_{S C M}\right|^{2}}>/ H_{H_{0}}{ }_{H_{1}} \lambda$

Asymptotically, $H_{1}:-N \ln \Lambda \rightarrow \chi_{m(m+1)}^{2}$.

Figure 1 shows the analyzed dataset in RGB coloring and the GLRT values, normalized (between 0 and 1). The estimation has been made over a moving $9 \times 9$ square window.

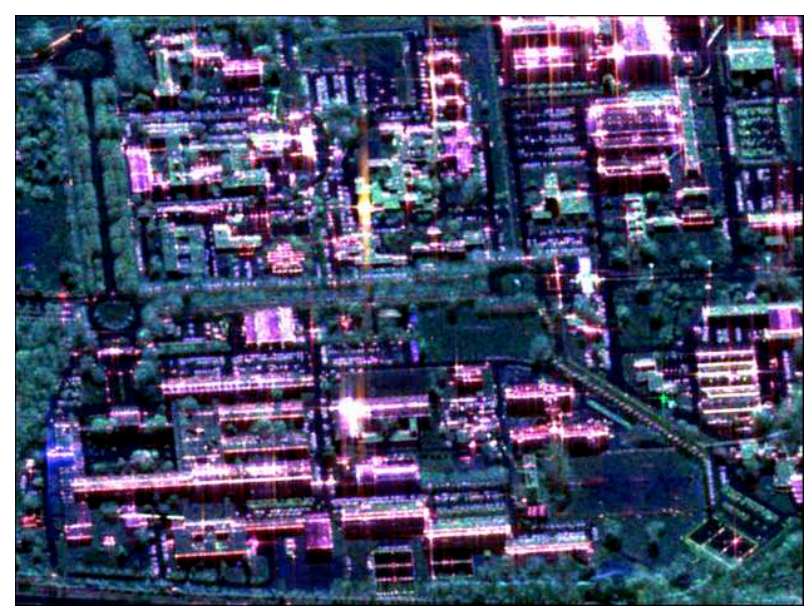

(a)

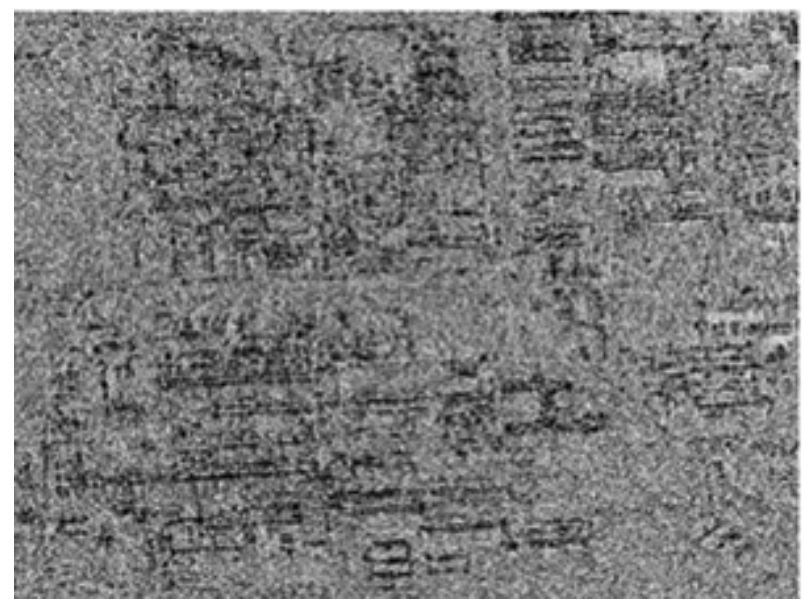

(b)

Figure 1: PolSAR dataset (a) and GLRT values (b) for the Toulouse site (Ramses, $\mathrm{X}$-band) 
The obtained results match expectations: i.e., those areas with distributed targets (such as forests and agricultural fields) expose not only Gaussian distribution, but also strong circularity. On the other hand, the GLRT values are small valued for urban areas and even the Gaussian distribution is not much confirmed.

\subsection{Spherically Invariant Random Processes}

In this case, the stochastic model is:

$$
p_{m}(\mathbf{k})=\int_{0}^{\infty} \frac{1}{(\pi \tau)^{m}|\mathbf{M}|} \exp \left(-\frac{\mathbf{k}^{H} \mathbf{M}^{-1} \mathbf{k}}{\tau}\right) p(\tau) d \tau=\frac{h_{m}\left(\mathbf{k}^{H} \mathbf{M}^{-1} \mathbf{k}\right)}{\pi^{m}|\mathbf{M}|}
$$

With the previous notations, the sphericity test makes use of the GLRT adjusted for CES (Complex Elliptically Symmetric) distributions [7], [8], known as aGLRT.

The hypotheses remain the same and the sphericity test becomes:

$$
\Lambda\left(\mathbf{k}_{1}, \ldots, \mathbf{k}_{N}\right)=\frac{\max _{\mathbf{R}, \mathbf{C}=\mathbf{0}_{m}}\left\{p_{\mathbf{k}}\left(\mathbf{k}_{1}, \ldots, \mathbf{k}_{N}\right)\right\}}{\max _{\mathbf{R}}\left\{p_{\mathbf{k}}\left(\mathbf{k}_{1}, \ldots, \mathbf{k}_{N}\right)\right\}}=\frac{|\mathbf{R}| H_{1}}{|\mathbf{T}|^{2}}>/{ }_{H_{0}} \lambda
$$

Asymptotically, $\operatorname{GLRT}\left(\mathbf{R}_{M L}\right)$ is equivalent to $\operatorname{aGLRT}\left(\mathbf{R}_{S C M}\right)$ under the $\mathrm{H}_{1}$ hypothesis:

$$
\Lambda_{a}\left(\mathbf{k}_{1}, \ldots, \mathbf{k}_{N}\right)=-3(N-m) \ln \left(\frac{\left|\hat{\mathbf{R}}_{S C M}\right|}{\left|\hat{\mathbf{T}}_{S C M}\right|^{2}} \frac{1}{\hat{\gamma}}\right)>{ }_{H_{0}}^{H_{1}} \lambda_{a}
$$

Finally, it is also possible to show that $\mathrm{H}_{1}: \Lambda_{a}\left(\mathbf{k}_{1}, \ldots, \mathbf{k}_{N}\right) \rightarrow \chi_{m}^{2}$.

Figure 11 shows the detection map of non-circular random processes, obtained for an imposed false alarm rate $P_{F A}=10^{-2}$ and a moving analysis $9 \times 9$ square window.

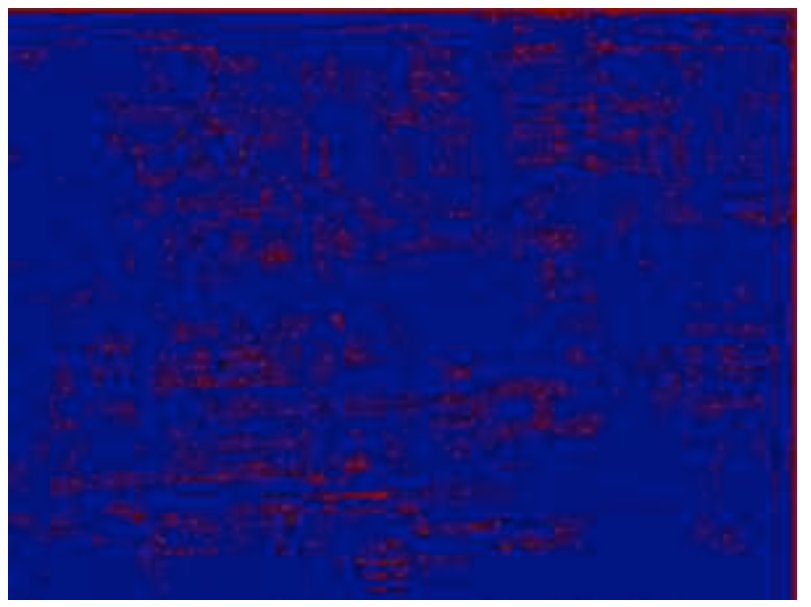

Figure 2: Detection map of non-circularity for the Toulouse dataset (Ramses, bande $\mathrm{X}$ )
The result confirms once again our expectations, as the strongly non-circular regions are located in urban areas.

\section{CONCLUSIONS}

Obtained results show that circularity assumption is generally respected for the considered datasets. This is even more valid for those areas with uniform and distributed targets (forests, agricultural fields, etc.). However, the urban regions with high density of point scatterrers and quite heterogeneous are less suited form the circularity point of view.

The bottom line is that characterization of urban regions is much more complex (and difficult) - since a more complex model, with more parameters, is required. In practice, circularity is often assumed and most of the developed models (the SIRV model for one) bear this limitation. In the light of the results shown in this paper, those models are less appropriate for urban areas characterization.

However, alternative explications are possible. As an example, the root of this inappropriateness might as well be the assumed ergodicity / stationarity (in spatial sense) for the backscattered signal and, also, even in the hypothesis of randomness: targets exhibit a deterministic behavior.

First, the very use of a sliding analysis window for estimating the stochastic parameters of the scattered signal may be questioned, as it implicitly assume that the considered signal is ergodic / stationary. While this hypothesis holds for distributed and uniform targets, where the physical parameters (and, thus, the electromagnetic scattering behavior) differs very little from one resolution cell to another, in urban areas the physical structure (and, as such, its electromagnetic behavior) may change considerably from one resolution cell to the next. This makes the hypothesis of ergodicity / stationarity less applicable.

Second, one should not that even the randomness of the radar echo is not given, but assumed. This is mainly a way to deal with the inherent complexity of the signal. Anyway, for identical measuring conditions, the recorded radar data is perfectly identical. Even if small differences in measuring conditions lead to strong discrepancies in the recorded data, this is not an evidence for randomness, as such behavior can be fully explain under a deterministic paradigm - the chaotic models.

Various parameters, such as meteorological conditions and, even more important, the changes that the target suffers in time (between two succeeding acquisitions, for example), account for the observed randomness of the recorded data.

However, these changes of the target are more significant for green targets (such as forests and agricultural fields), where humidity and wind modify both their physical structure and their electromagnetic behavior. On the other hand, those changes are less significant for urban targets and, as such, randomness is less likely for the latter. 
In perspective, applying chaotic [9], [11], [12] [13] or pseudo-chaotic [10] models to PolSAR / InSAR data from urban areas is envisageable. These models should be able to take into account the deterministic features of those areas (presence of dihedral angles, straight edges, cavities, etc.), while still leaving room for some unpredictability (orientation of those elements).

Using chaotic models in PolSAR and InSAR data will make the object of future work.

\section{ACKNOWLEDGEMENT}

The authors would also like to thank Dr. C. Tison (CNES, France) for providing the high-resolution POLSAR images over Toulouse.

\section{REFERENCES}

[1] L. Bombrun, G. Vasile, M. Gay, F.-C. Totir, "Hierarchical segmentation of polarimetric SAR images using heterogeneous clutter models", IEEE Transactions on Geoscience and Remote Sensing, vol. 49, no. 2, pp. 726-737, 2011.

[2] B. Picinbono, "On circularity", IEEE Transactions on signal processing, vol. 42, no. 12, pp. 3473-3482, 1994.

[3] E. Ollila, J. Eriksson, V. Koivunen, "Complex elliptically symmetric random variables - generation, characterization, and circularity tests", IEEE Transactions on Signal Processing, vol. 59, no. 1, pp. 58-69, 2011.

[4] F. D. Neeser, J. L. Massey, "Proper complex random processes with applications to information theory", IEEE Transactions on Information Theory, vol. 39, no. 4, pp. 1293-1302,1993.

[5] L. L. Scharf, C. T. Mullis, "Canonical coordinates and the geometry of inference, rate, and capacity", IEEE Transactions on Signal Processing, vol. 48, no. 3, pp. 824-831, 2000.

[6] A. T. Walden, P. Rubin-Delanchy, "On testing for impropriety of complex-valued gaussian vectors", IEEE Transactions on Signal Processing, vol. 57, no. 3, pp. 835-842, 2009.

[7] D. E. Tyler, "Robustness and efficiency of scatter matrices", Biometrika, vol. 70, pp. 411-420, 1983.

[8] E. Ollila, V. Koivunen, "Adjusting the generalized likelihood ratio test of circularity robust to non-normality", IEEE Workshop on Signal Process. Adv. Wireless Commun, Italy, 2009, pp. 558562 .

[9] J. B. Gao, Z. M. Zheng, "Direct dynamical test for deterministic chaos and optimal embedding of a chaotic time series", Physical Review E 49, (article 3807), 1994.

[10] J. B. Gao, W.W. Tung, Y.H. Cao, J. Hu, Y. Qi, "Power-law sensitivity to initial conditions in atime series with applications to epileptic seizure detection", Physica A no. 353, pp. 613-624, 2005.

[11] S. Haykin, S. Puthusserypady, "Chaotic dynamics of sea clutter", Chaos, no. 7, pp. 777-802, 1997.
[12] S. Haykin, Chaotic dynamics of sea clutter, John Wiley, 1999.

[13] J. Hu, J. Gao, K. Yao, "Power-law sensitivity to initial conditions in sea clutter", Radar Conference, 2005 IEEE International Volume, Issue 9-12, pp. 956 - 961, May 2005.

[14] B. Picinbono, "Second-order complex random vectors and normal distributions", IEEE Transactions on Signal Processing, vol. 44, pp. 2637-2640, 1996.

[15] E. Ollila, J. Eriksson, V. Koivunen, "Complex elliptically symmetric random variables - generation, characterization, and circularity tests", IEEE Transactions on Signal Processing, vol. 59, no. 1, pp. 58-69, January 2011. 